\title{
Boundary triples for integral systems on finite intervals
}

\author{
DMYTRO STRELNIKOV
}

(Presented by M.M. Malamud)

\begin{abstract}
Let $P, Q$ and $W$ be real functions of bounded variation on $[0, l]$ and let $W$ be nondecreasing. The following integral system

$$
J \vec{f}(x)-J \vec{a}=\int_{0}^{x}\left(\begin{array}{cc}
\lambda d W-d Q & 0 \\
0 & d P
\end{array}\right) \vec{f}(t), \quad J=\left(\begin{array}{cc}
0 & -1 \\
1 & 0
\end{array}\right)
$$

on a finite compact interval $[0, l]$ has been studied in [6]. A maximal and a minimal linear relation $A_{\max }$ and $A_{\min }$ associated with the integral system (9) are studied in the Hilbert space $L^{2}(W)$. It is shown that the linear relation $A_{\min }$ is symmetric with deficiency indices $n_{ \pm}\left(A_{\min }\right)=2$ and $A_{\max }=A_{\min }^{*}$. Boundary triples for $A_{\max }$ are constructed and the corresponding Weyl functions are calculated.
\end{abstract}

2010 MSC. Primary 34B20; Secondary 45A05; 47A06.

Key words and phrases. Integral system, boundary triple, symmetric linear relation, deficiency indices, Weyl function.

\section{Introduction}

This paper focuses on the following integral system

$$
J \vec{f}(x)-J \vec{a}=\int_{0}^{x} d S(t) \cdot \vec{f}(t)
$$

where $J$ and $d S$ are $2 \times 2$ matrices of the form:

$$
J=\left(\begin{array}{cc}
0 & -1 \\
1 & 0
\end{array}\right), \quad d S=\left(\begin{array}{cc}
\lambda d W-d Q & 0 \\
0 & d P
\end{array}\right),
$$

Received 12.09.2017

The author is grateful to professor V. Derkach for constant attention to this work. 
$\lambda \in \mathbb{C}$, all functions $P, Q$ and $W$ are real of bounded variation on $[0, l]$ and $W$ is nondecreasing on $[0, l]$. Such systems were studied in $[2,3,6]$. System (1.1) contains Sturm-Liouville systems, Stieltjes string and Krein-Feller string $[13,18]$ as special cases.

We associate with system (1.1) minimal $A_{\min }$ and maximal $A_{\max }$ linear relations. In contrast to the Sturm-Liouville case $A_{\min }$ and $A_{\max }$ may be multivalued, therefore we use for them a term linear relation (see [1]). It turns out that the linear relation $A_{\min }$ is symmetric with deficiency indices $(2,2)$.

The notions of the boundary triple and Weyl function introduced in $[7,8,19]$ and $[10]$, respectively, were proved to be useful in the study of spectral problems and extension theory problems for symmetric operators, see $[11,12,14]$. Boundary triples for various differential and difference operators were constructed in $[4,10,11,14,19,21,22]$.

A boundary triple for the linear relation $A_{\max }$ is constructed in the paper and the corresponding matrix Weyl function is calculated. In a similar way some intermediate extensions of the linear relation $A_{\text {min }}$ with deficiency indices $(1,1)$ are considered and their scalar Weyl functions are found.

\section{Preliminaries}

\subsection{Linear relations}

Let $\mathfrak{H}$ be a Hilbert space. Any linear supspace of $\mathfrak{H} \times \mathfrak{H}$ is called a linear relation in $\mathfrak{H},[1]$.

The domain, the range, the kernel, and the multivalued part of a linear relation $T$ are defined by the following equalities (see $[1,5]$ ):

$$
\begin{aligned}
\operatorname{dom} T: & =\left\{f:\left(\begin{array}{l}
f \\
g
\end{array}\right) \in T\right\}, & \operatorname{ran} T:=\left\{g:\left(\begin{array}{l}
f \\
g
\end{array}\right) \in T\right\}, \\
\operatorname{ker} T: & =\left\{f:\left(\begin{array}{l}
f \\
0
\end{array}\right) \in T\right\}, & \operatorname{mul} T:=\left\{g:\left(\begin{array}{l}
0 \\
g
\end{array}\right) \in T\right\} .
\end{aligned}
$$

The adjoint linear relation $T^{*}$ is defined by

$$
T^{*}:=\left\{\left(\begin{array}{l}
u \\
v
\end{array}\right) \in \mathfrak{H} \times \mathfrak{H}:(v, f)_{\mathfrak{H}}=(u, g)_{\mathfrak{H}} \text { for some }\left(\begin{array}{l}
f \\
g
\end{array}\right) \in T\right\} .
$$

A linear relation $T$ in $\mathfrak{H}$ is called closed if $T$ is closed as a subspace of $\mathfrak{H} \times \mathfrak{H}$. The set of all closed linear operators (relations) is denoted by $\mathcal{C}(\mathfrak{H})(\widetilde{\mathcal{C}}(\mathfrak{H}))$. Identifying a linear operator $T \in \mathcal{C}(\mathfrak{H})$ with its graph one can consider $\mathcal{C}(\mathfrak{H})$ as a part of $\widetilde{\mathcal{C}}(\mathfrak{H})$. 
Definition 2.1. Suppose $T$ is a linear relation, $\lambda \in \mathbb{C}$ then

$$
T-\lambda I:=\left\{\left(\begin{array}{c}
f \\
g-\lambda f
\end{array}\right):\left(\begin{array}{l}
f \\
g
\end{array}\right) \in T\right\} .
$$

A point $\lambda \in \mathbb{C}$ such that $\operatorname{ker}(T-\lambda I)=\{0\}$ and $\operatorname{ran}(T-\lambda I)=\mathfrak{H}$ is called a regular point of the linear relation $T$ and is written $\lambda \in \rho(T)$.

The point spectrum and the continuous spectrum of the linear relation $T$ are defined by

$$
\begin{gathered}
\sigma_{p}(T):=\{\lambda \in \mathbb{C}: \operatorname{ker}(T-\lambda I) \neq\{0\}\} \\
\sigma_{c}(T):=\left\{\lambda \in \mathbb{C}: \lambda \notin \sigma_{p}(T), \operatorname{ran}(T-\lambda I) \neq \overline{\operatorname{ran}(T-\lambda I)}=\mathfrak{H}\right\} .
\end{gathered}
$$

For $\lambda \in \mathbb{C}_{ \pm}$let us set $\mathfrak{N}_{\lambda}(T):=\operatorname{ker}\left(T^{*}-\lambda I\right)$ and

$$
\hat{\mathfrak{N}}_{\lambda}(T):=\left\{\left(\begin{array}{c}
f_{\lambda} \\
\lambda f_{\lambda}
\end{array}\right): f_{\lambda} \in \mathfrak{N}_{\lambda}\right\}
$$

A linear relation $A$ is called symmetric if $A \subseteq A^{*}$. The deficiency indices of a symmetric linear relation $A$ are defined by

$$
n_{ \pm}(A):=\operatorname{dim} \operatorname{ker}\left(A^{*} \mp i I\right)
$$

\subsection{Boundary triples}

In the case of densely defined operators a boundary triple notion was introduced in $[7,8,14,19]$ (in different forms). Following the paper [21] we shall give a general definition of a boundary triple for the linear relation $T$.

Definition 2.2. The tuple $\Pi=\left\{\mathcal{H}, \Gamma_{0}, \Gamma_{1}\right\}$ where $\mathcal{H}$ is a Hilbert space, $\Gamma_{0}$ and $\Gamma_{1}$ are linear mappings from $T$ to $\mathcal{H}$ is called a boundary triple for linear relation $T$, if the following conditions hold:

(i) generalized Green's identity

$$
(g, u)_{\mathfrak{H}}-(f, v)_{\mathfrak{H}}=\left(\Gamma_{1}\left(\begin{array}{l}
f \\
g
\end{array}\right), \Gamma_{0}\left(\begin{array}{l}
u \\
v
\end{array}\right)\right)_{\mathcal{H}}-\left(\Gamma_{0}\left(\begin{array}{l}
f \\
g
\end{array}\right), \Gamma_{1}\left(\begin{array}{l}
u \\
v
\end{array}\right)\right)_{\mathcal{H}}
$$

holds for all $\left(\begin{array}{l}f \\ g\end{array}\right),\left(\begin{array}{l}u \\ v\end{array}\right) \in T$;

(ii) the mapping $\Gamma=\left(\begin{array}{c}\Gamma_{0} \\ \Gamma_{1}\end{array}\right): T \rightarrow \mathcal{H} \times \mathcal{H}$ is surjective. 
If the linear relation $T$ is adjoint to some symmetric linear relation $A$ then there exists a boundary triple for $T$ if and only if the deficiency indices of $A$ coincide $\left(n_{+}(A)=n_{-}(A)\right)$, see $[11,19,21]$.

An extension $\widetilde{A}$ of a symmetric linear relation $A$ is called proper if $A \subsetneq \widetilde{A} \subsetneq A^{*}$. The class of all proper extensions of the linear relation $A$ completed with relations $A$ and $A^{*}$ is denoted by $\operatorname{Ext}(A)$. Denote also

$$
A_{\Theta}:=\left\{\left(\begin{array}{l}
f \\
g
\end{array}\right) \in A^{*}: \Gamma\left(\begin{array}{l}
f \\
g
\end{array}\right) \in \Theta\right\} .
$$

Proposition 2.3. [11] Let $A$ be a symmetric linear relation, $\Pi=\left\{\mathcal{H}, \Gamma_{0}, \Gamma_{1}\right\}$ be a boundary triple for the adjoint linear relation $A^{*}$. Then the mapping $\Gamma: \widetilde{A}=A_{\Theta} \rightarrow \Theta=\Gamma \widetilde{A}$ is a one-to-one mapping from $\operatorname{Ext}(A)$ to $\widetilde{\mathcal{C}}(\mathfrak{H})$. Notice also that $A_{\Theta}$ is selfadjoint if and only if the linear relation $\Theta$ is selfadjoint.

In particular, linear relations

$$
A_{0}:=\operatorname{ker} \Gamma_{0}, \quad A_{1}:=\operatorname{ker} \Gamma_{1}
$$

are disjoint, i.e. $A_{0} \cap A_{1}=A$, and they are selfadjoint extensions of the symmetric linear relation $A$ (see [11]).

Suppose $A$ is adjoint for the linear relation $T$ from Definition 2.2 The conditions ensuring the symmetry of $A$ are provided by the next theorem. In the case of single-valued linear operator $T$ the corresponding theorem was proved in [12].

Theorem 2.4. [12] Let $T$ be a linear relation in the Hilbert space $\mathfrak{H}$, $\Pi=\left\{\mathcal{H}, \Gamma_{0}, \Gamma_{1}\right\}$ be its boundary triple such that $n:=\operatorname{dim} \mathcal{H}<\infty$ and $A=\operatorname{ker} \Gamma$. If the following conditions hold:

(i) $\operatorname{ran} T=\mathfrak{H}$;

(ii) $\operatorname{dim} \operatorname{ker} T=n$ and $\operatorname{ker} A=\{0\}$,

then linear relations $A, T$ are closed, $T=A^{*}$ and $n_{+}(A)=n_{-}(A)=n$.

Definition 2.5. $[10,11]$ Let $\Pi=\left\{\mathcal{H}, \Gamma_{0}, \Gamma_{1}\right\}$ be a boundary triple for linear relation $A^{*}$. Operator valued functions $M(\cdot), \gamma(\cdot)$ defined by

$$
M(\lambda) \Gamma_{0} \hat{f}_{\lambda}=\Gamma_{1} \hat{f}_{\lambda}, \quad \gamma(\lambda) \Gamma_{0} \hat{f}_{\lambda}=f_{\lambda}, \quad \hat{f}_{\lambda} \in \hat{\mathfrak{N}}_{\lambda}, \quad \lambda \in \rho\left(A_{0}\right)
$$

are called the Weyl function and the $\gamma$-field of the symmetric linear relation $A$ with respect to the boundary triple $\Pi$.

Definition 2.6. An operator valued function $F: \mathbb{C}_{+} \cup \mathbb{C}_{-} \rightarrow \mathcal{B}(\mathcal{H})$ is said to belong to the class $R[\mathcal{H}]$ if the following conditions hold: 
(i) $F$ is holomorphic in $\mathbb{C}_{+} \cup \mathbb{C}_{-}$;

(ii) $\operatorname{Im} F(\lambda) \geq 0$ as $\lambda \in \mathbb{C}_{+}$;

(iii) $F(\bar{\lambda})=F^{*}(\lambda), \quad \lambda \in \mathbb{C}_{+} \cup \mathbb{C}_{-}$.

If $\mathcal{H}=\mathbb{C}$ then $R[\mathcal{H}]$ is denoted by $R$.

It is known that the Weyl function $M(\lambda)$ of a linear relation $A$ from Definition 2.5 belongs to the class $R[\mathcal{H}]$. The next proposition gives a description of the spectrum of a linear $\widetilde{A} \in \operatorname{Ext}(A)$.

Proposition 2.7. [11] Let $A$ be a symmetric linear relation in $\mathfrak{H}, \Pi=$ $\left\{\mathcal{H}, \Gamma_{0}, \Gamma_{1}\right\}$ be a boundary triple for $A^{*}, M(\lambda)$ be the corresponding Weyl function of $A, \Theta \in \widetilde{\mathcal{C}}(\mathcal{H})$, and $\lambda \in \rho\left(A_{0}\right)$. Then:

(i) $\lambda \in \rho\left(\widetilde{A}_{\Theta}\right) \Longleftrightarrow 0 \in \rho(\Theta-M(\lambda))$;

(ii) $\lambda \in \sigma_{p}\left(\widetilde{A}_{\Theta}\right) \Longleftrightarrow 0 \in \sigma_{p}(\Theta-M(\lambda))$.

\subsection{Integral systems}

Let us consider on a compact interval $[0, l]$ an integral system

$$
J \vec{f}(x)-J \vec{a}(x)=\int_{0}^{x} d S(t) \cdot \vec{f}(t)
$$

where $\vec{f}$ is a $n \times 1$ complex vector, $\vec{a}$ is a fixed complex vector valued function of bounded variation, $d S$ is a finite $n \times n$ measure, and $J$ is a constant $n \times n$ matrix such that $J^{*}=-J$.

Definition 2.8. We say that a vector valued function $\vec{f}$ is a solution of integral system (2.13) if (each component of) $\vec{f}$ is of bounded variation and the equality (2.13) holds for every point of $[0, l]$.

It is easy to see that if for some vector valued function $\vec{f}$ the righthand part of equality (2.13) exists for all $x \in[0, l]$ then it is of bounded variation on $[0, l]$ and therefore inclusion $\vec{f} \in B V[0, l]$ is necessery for (2.13). The same condition is also sufficient for existence of the integral in the right-hand part of (2.13) (as a Lebesgue-Stietjes integral).

In general case measure $d S$ is not supposed to be absolutely continuous and may have mass points on $[0, l]$. Therefore in equality $(2.13)$ and in the following we should understand $\int_{a}^{b} f d \mu$ as the Lebesgue-Stieltjes integral $\int f \chi_{[a, b)} d \mu$, where $\chi_{[a, b)}$ is the characteristic function of the halfopen interval. Under this conventions integrals as functions of its limits of integration are left-continuous.

The following theorem was proved in [6]. 
Theorem 2.9. [6] For any left-continuous vector-function $\vec{a}(x) \in B V[0, l]$ there exists a unique solution of (2.13).

Further in this paper the integration by parts formula will be used in the following form (see [15]). If $u$ is a left-continuous function of bounded variation then we denote by $u_{+}$the right-continuous function that coincides with $u$ in every continuity point. If $v$ is another left-continuous function of bounded variation then the following equality holds

$$
\int_{y}^{x} v d u=v(x) u(x)-v(y) u(y)-\int_{y}^{x} u_{+} d v .
$$

Now suppose that $n=2$, matrices $J$ and $d S$ have the following form

$$
J=\left(\begin{array}{cc}
0 & -1 \\
1 & 0
\end{array}\right), \quad d S=\left(\begin{array}{cc}
\lambda d W-d Q & 0 \\
0 & d P
\end{array}\right)
$$

where $\lambda$ is a complex parameter, $P, Q$ and $W$ are of bounded variation and left-continuous on $[0, l]$ functions that satisfy the condition

$$
P(0)=Q(0)=W(0)=0
$$

and $W$ is nondecreasing. We assume that functions $P, Q$ and $W$ are defined on the whole real line and their values on the intervals $(-\infty, 0]$ and $[l,+\infty)$ are constant.

In the remaining part of this paper attention will be restricted to considering (2.13) when the matrices $J$ and $d S$ have the form (2.15).

Everywhere in the following we use

Assumption 2.10. Functions $Q$ and $W$ have no common discontinuities with $P$.

\section{Green's identity and linear relation $A_{\max }$}

\subsection{Green's identity}

Let $\mathcal{L}^{2}(W)$ be an inner product space, which consists of complex valued functions $f$ such that

$$
\int_{0}^{l}|f(t)|^{2} d W(t)<\infty .
$$

The inner product in $\mathcal{L}^{2}(W)$ is defined by

$$
(f, g)_{W}=\int_{0}^{l} f(t) \overline{g(t)} d W(t) .
$$


Denote by $L^{2}(W)$ the corresponding quotient space, which consists of equivalence classes with respect to the measure $d W$. To avoid confusion we will denote elements of the space $L^{2}(W)$ by gothic letters $\mathfrak{f}, \mathfrak{g}$ etc.

Let us consider the inhomogeneous system

$$
\left.J\left(\begin{array}{c}
f \\
f^{[1]}
\end{array}\right)\right|_{0} ^{x}=\int_{0}^{x}\left(\begin{array}{cc}
-d Q(t) & 0 \\
0 & d P(t)
\end{array}\right)\left(\begin{array}{c}
f \\
f^{[1]}
\end{array}\right)+\int_{0}^{x}\left(\begin{array}{cc}
d W(t) & 0 \\
0 & 0
\end{array}\right)\left(\begin{array}{l}
g \\
0
\end{array}\right) .
$$

Definition 3.1. A pair $\{\vec{f}, g\}$ that consists of a vector-function $\vec{f}=$ $\left(\begin{array}{c}f \\ f^{[1]}\end{array}\right)$ and a scalar function $g$ is said to satisfy system (3.3) (or that $\vec{f}$ is a solution of this system with fixed $g$ ), if the following conditions hold:

(i) $g \in \mathcal{L}^{2}(W)$;

(ii) $\vec{f} \in B V[0, l]$;

(iii) the equality (3.3) holds for each $x \in[0, l]$.

Remark. It is clear that condition $\vec{f} \in B V[0, l]$ is automatically satisfied as equality (3.3) holds. In this case it follows from $\vec{f} \in B V[0, l]$ that $f \in \mathcal{L}^{2}(W)$.

The componentwise rewriting of system (3.3) gives

$$
\left\{\begin{array}{l}
f(x)-f(0)=\int_{0}^{x} f^{[1]}(t) d P(t), \\
f^{[1]}(x)-f^{[1]}(0)=\int_{0}^{x}(f(t) d Q(t)-g(t) d W(t)) .
\end{array}\right.
$$

Theorem 3.2 (The first Green's identity). Suppose that Assumption 2.10 holds and pairs $\{\vec{f}, g\},\{\vec{u}, v\}$ satisfy system (3.3) (see Definition 3.1). Then for any $\alpha, \beta \in[0, l]$ the next equality holds

$$
\int_{\alpha}^{\beta} g u d W=\int_{\alpha}^{\beta} f u d Q+\int_{\alpha}^{\beta} f^{[1]} u^{[1]} d P-\left.f^{[1]} u\right|_{\alpha} ^{\beta} .
$$

Proof. From (3.4) we have:

$$
d u=u^{[1]} d P, \quad d f^{[1]}=f d Q-g d W .
$$

It follows from Assumtion 2.10 that functions $u$ and $f^{[1]}$ have no common discontinuities. Consider the measure $d\left(f^{[1]} u\right)$. Then

$$
d\left(f^{[1]} u\right)=d f^{[1]} u+f^{[1]} d u=f u d Q+f^{[1]} u^{[1]} d P-g u d W
$$


hence

$$
g u d W=f u d Q+f^{[1]} u^{[1]} d P-d\left(f^{[1]} u\right) .
$$

To conclude the proof it remains to note that function $f^{[1]} u$ is leftcontinuous and to integrate equality $(3.8)$ over $[\alpha, \beta)$.

For a pair of vector valued functions $\vec{f}=\left(\begin{array}{c}f \\ f^{[1]}\end{array}\right)$ and $\vec{u}=\left(\begin{array}{c}u \\ u^{[1]}\end{array}\right)$ we define the generalized Wronskian by

$$
[\vec{f}, \vec{u}]:=\left(f u^{[1]}-f^{[1]} u\right)
$$

Theorem 3.3. Suppose Assumption 2.10 holds and pairs $\{\vec{f}, g\},\{\vec{u}, v\}$ satisfy system (3.3). Then for any $\alpha, \beta \in[0, l]$ the next equality holds

$$
\int_{\alpha}^{\beta}(g u-f v) d W=\left.[\vec{f}, \vec{u}]\right|_{\alpha} ^{\beta}
$$

Proof. Application of Theorem 3.2 gives

$$
\begin{aligned}
& g u d W=f u d Q+f^{[1]} u^{[1]} d P-d\left(f^{[1]} u\right), \\
& f v d W=f u d Q+f^{[1]} u^{[1]} d P-d\left(f u^{[1]}\right) .
\end{aligned}
$$

Subtraction of (3.12) from (3.11) proves the statement.

Corollary 3.4 (The second Green's identity). For any two pairs $\{\vec{f}, g\}$ and $\{\vec{u}, v\}$ satisfying (3.3) the generalized Green's identity holds

$$
(g, u)_{W}-(f, v)_{W}=\left(\left.f^{[1]} \bar{u}\right|_{0}-\left.f^{[1]} \bar{u}\right|_{l}\right)-\left(\left.f \overline{u^{[1]}}\right|_{0}-\left.f \overline{u^{[1]}}\right|_{l}\right) .
$$

\subsection{Linear relation $A_{\max }$}

Definition 3.5. We shall say that a pair of classes $\left(\begin{array}{l}\mathfrak{f} \\ \mathfrak{g}\end{array}\right) \in L^{2}(W) \times$ $L^{2}(W)$ belongs to the linear relation $A_{\max }$ if there exist functions $f, f^{[1]}$ and $g$ such that

(i) the pair $\{\vec{f}, g\}$, where $\vec{f}=\left(\begin{array}{c}f \\ f^{[1]}\end{array}\right)$, satisfies (3.3) (in the sense of Definition 3.1);

(ii) $f \in \mathfrak{f}, g \in \mathfrak{g}$. 
In the succeeding we require the following

Assumption 3.6. For any $a, b, a_{1}, b_{1} \in \mathbb{C}$ there exists a pair $\{\vec{f}, g\}$ satisfying (3.3) such that

$$
f(0)=a, \quad f^{[1]}(0)=a_{1}, \quad f(l)=b, \quad f^{[1]}(l)=b_{1} .
$$

In particular, if $d Q \equiv 0$ then a sufficient condition for Assumption 3.6 to hold is the next

Proposition 3.7. Suppose $d Q \equiv 0$. If there exist closed on the left and disjoint intervals $i_{1}$ and $i_{2}$ on $[0, l]$ such that

$$
\begin{aligned}
\operatorname{dim} L^{2}\left(i_{j}, W\right) & >0 \quad(j \in\{1,2\}), \\
\frac{1}{d W\left(i_{2}\right)} \int_{i_{2}} P(t) d W(t) & >\frac{1}{d W\left(i_{1}\right)} \int_{i_{1}} P(t) d W(t),
\end{aligned}
$$

then Assumption 3.6 holds.

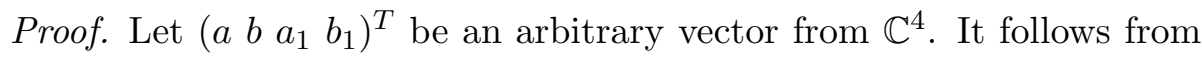
condition (3.15) that there exist functions $u_{j}$ that equal to one on interval $i_{j}$ and equal zero on its complement, and $\left\|u_{j}\right\|_{W}=d W\left(i_{j}\right) \neq 0(j \in$ $\{1,2\})$.

Put $g=c_{1} u_{1}+c_{2} u_{2}$, where $c_{1}$ and $c_{2}$ are some constants from $\mathbb{C}$. We shall define vector-function $\vec{f}$ by the next system

$$
\left\{\begin{array}{l}
f(x)=a+\int_{0}^{x} f^{[1]}(t) d P(t), \\
f^{[1]}(x)=a_{1}-\int_{0}^{x} g(t) d W(t) .
\end{array}\right.
$$

It is clear that for any $c_{1}, c_{2} \in \mathbb{C}$ we have $g \in \mathcal{L}^{2}(W)$. Further, it follows from system (3.17) that vector-function $\vec{f}$ is of bounded variation on $[0, l]$ and $\vec{f}(0)=\left(a a_{1}\right)^{T}$, i.e. the pair $\{\vec{f}, g\}$ satisfies system (3.3) with the initial conditions given in advance.

Let us show now that constants $c_{1}$ and $c_{2}$ may be chosen so that equality $\vec{f}(l)=\left(\begin{array}{ll}b & b_{1}\end{array}\right)^{T}$ holds. It is true if and only if there exists a solution of the next system (with respect to $c_{1}, c_{2}$ )

$$
\left\{\begin{array}{l}
c_{1} d W\left(i_{1}\right)+c_{2} d W\left(i_{2}\right)=a_{1}-b_{1}, \\
c_{1} \int_{0}^{l} d P(t) \int_{0}^{t} u_{1}(s) d W(s)+c_{2} \int_{0}^{l} d P(t) \int_{0}^{t} u_{2}(s) d W(s)= \\
\end{array}\right.
$$


By Assumption 2.10 functions $P$ and $W$ have no common discontinuities, so using integration by parts formula (2.14) we get:

$$
\int_{0}^{l} d P(t) \int_{0}^{t} u_{j}(s) d W(s)=P(l) d W\left(i_{j}\right)-\int_{0}^{l} P(t) u_{j}(t) d W(t)
$$

where $j \in\{1,2\}$. Multiplying the first equation of system (3.18) by $P(l)$ and subtracting it from the second one and combining the obtained equation with (3.19) we will have a system (with respect to $c_{1}, c_{2}$ ), whose determinant

$$
\left|\begin{array}{ll}
d W\left(i_{1}\right) & d W\left(i_{2}\right) \\
\int_{i_{1}} P(t) d W(t) & \int_{i_{2}} P(t) d W(t)
\end{array}\right|
$$

is strictly positive due to (3.16). This ensures the solvability of system (3.18).

Theorem 3.8. Let Assumption 2.10 and Assumption 3.6 be satisfied and let the mappings $\Gamma_{0}, \Gamma_{1}: A_{\max } \rightarrow \mathbb{C}^{2}$ be defined by

$$
\Gamma_{0}\left(\begin{array}{l}
\mathfrak{f} \\
\mathfrak{g}
\end{array}\right):=\left(\begin{array}{c}
f(0) \\
f(l)
\end{array}\right), \quad \Gamma_{1}\left(\begin{array}{l}
\mathfrak{f} \\
\mathfrak{g}
\end{array}\right):=\left(\begin{array}{c}
f^{[1]}(0) \\
-f^{[1]}(l)
\end{array}\right)
$$

where the pair $\{\vec{f}, g\}$ satisfies system (3.3), $f \in \mathfrak{f}, g \in \mathfrak{g}$. Then:

(i) the mappings $\Gamma_{0}, \Gamma_{1}$ are well-defined;

(ii) the tuple $\left\{\mathbb{C}^{2}, \Gamma_{0}, \Gamma_{1}\right\}$ is a boundary triple for the linear relation $A_{\max }$.

Proof. (i) Let us show first that the mappings $\Gamma_{0}, \Gamma_{1}$ from (3.21) are independent of the choice of $f, g$ from classes $\mathfrak{f}, \mathfrak{g}$ respectively. It is clear that if a pair $\left\{\vec{f}, g_{1}\right\}$ satisfies system (3.3) then a pair $\left\{\vec{f}, g_{2}\right\}$ also satisfies (3.3) if $g_{1}$ and $g_{2}$ are equivalet with respect to the measure $d W$. It means that the values of $\Gamma_{0}, \Gamma_{1}$ are independent of the choice of $g \in \mathfrak{g}$.

Further let us prove that the values of the mappings $\Gamma_{0}, \Gamma_{1}$ are independent of choosing an instance $f$ from the class $\mathfrak{f}$. Let pairs $\left\{\vec{f}_{1}, g\right\}$ and $\left\{\vec{f}_{2}, g\right\}$ satisfy system (3.3) such that $f_{1}, f_{2} \in \mathfrak{f}$. The application of Green's identity in the form (3.10) for both of the pairs on $[0, l]$ gives us two equalities. Subtracting one from the other gives

$$
0=\int_{0}^{l}\left(f_{2}-f_{1}\right) \bar{v} d W=\left.\left[\overrightarrow{f_{1}}-\overrightarrow{f_{2}}, \overrightarrow{\vec{u}}\right]\right|_{0} ^{l} .
$$


By Assumption 3.6 a pair of functions $\{\vec{u}, v\}$ satisfying system (3.3) can be chosen such that $u(0), u(l), u^{[1]}(0)$ and $u^{[1]}(l)$ may be arbitrary from $\mathbb{C}$. This means that we have

$$
\begin{aligned}
f_{1}(0) & =f_{2}(0), & f_{1}(l) & =f_{2}(l), \\
f_{1}^{[1]}(0) & =f_{2}^{[1]}(0), & f_{1}^{[1]}(l) & =f_{2}^{[1]}(l)
\end{aligned}
$$

which proofs that the mappings $\Gamma_{0}, \Gamma_{1}$ are single-valued.

(ii) It follows directly from Corollary 3.4 and Assumption 3.6 that the requirements of Definition 2.2 are satisfied.

Remark 3.9. Evidently, if Assumption 3.6 does not hold then the objects $\Gamma_{0}$ and $\Gamma_{1}$, defined by $(3.21)$, in general are not operators but linear relations in $L^{2}(W)^{2} \times \mathbb{C}^{2}$. Such boundary triples were considered in [9].

It is also possible that if Assumption 3.6 does not hold then the mapping $\Gamma=\left(\Gamma_{0} \Gamma_{1}\right)^{T}$ is not surjective. This happens, for example, if $d Q=0, d P=d x$, and $W$ is piecewise with a single jump.

In the case of $d Q \equiv 0$ system (3.4) can be rewritten as follows

$$
f(x)=f(0)+f^{[1]}(0) P(x)-\int_{0}^{x}\left\{\int_{0}^{t} g(s) d W(s)\right\} d P(t) .
$$

Function $G(t):=\int_{0}^{t} g(s) d W(s)$ is of bounded variation on $[0, l]$ and the set of its jumps is a subset of jumps of function $W$. Hence, functions $G$ and $P$ have no common discontinuities. The application of integration by parts formula (2.14) to equality (3.25) gives us (cf. [17, p. 650, equality $(1.1)])$

$$
f(x)=f(0)+f^{[1]}(0) P(x)-\int_{0}^{x}\{P(x)-P(t)\} g(t) d W(t) .
$$

This leads to the following

Proposition 3.10. Suppose Assumption 2.10 holds and $d Q \equiv 0$. Then the kernel of the linear relation $A_{\max } \subset L^{2}(W)^{2}$ is two-dimensional if and only if function $P$ is not equivalent to a constant in $L^{2}(W)$ and one-dimensional otherwise.

Proof. Let $g$ be zero element of $L^{2}(W)$. Then equality (3.26) takes the form

$$
f(x)=f(0)+f^{[1]}(0) P(x),
$$

which is equivalent to $f \in \operatorname{span}\{1, P\}$. 
Remark. Further, in the proof of Theorem 3.12 it will be shown that the kernel of linear relation $A_{\max }$ is always two-dimensional if in addition Assumption 3.6 holds.

Definition 3.11. We shall say that an element $\left(\begin{array}{l}\mathfrak{f} \\ \mathfrak{g}\end{array}\right)$ of the linear relation $A_{\text {max }}$ belongs to the linear relation $A_{\text {min }}$, if

$$
f(0)=f^{[1]}(0)=f(l)=f^{[1]}(l)=0 .
$$

It follows from equality (3.13) that the linear relation $A_{\min }$ is symmetric.

Theorem 3.12. Linear relations $A_{\min }$ and $A_{\max }$ are closed, $A_{\min }^{*}=$ $A_{\max }$, and deficiency indices of $A_{\min }$ are $(2,2)$.

Proof. We shall check that for linear relations $A_{\min }$ and $A_{\max }$ conditions of Theorem 2.4 are satisfied. It follows directly from Theorem 2.9 that $\operatorname{ran} A_{\max }=L^{2}(W)$. Let $\mathfrak{g}$ be an arbitrary class from $L^{2}(W), g$ be some instance of $\mathfrak{g}$. Then (for any fixed initial value) by Theorem 2.9 there exists a vector-function $\vec{f}=\left(\begin{array}{c}f \\ f^{[1]}\end{array}\right)$ such that pair $\{\vec{f}, g\}$ satisfies system (3.3) and, as a consequence, $\left(\begin{array}{l}\mathfrak{f} \\ \mathfrak{g}\end{array}\right) \in A_{\max }$.

Further, let us show that $\operatorname{dim} \operatorname{ker} A_{\max }=2$. By Theorem 2.9 if $g=0$ then for any complex numbers $a, a_{1}$ there exists a unique vector-function $\vec{f}$ such that $f(0)=a, f^{[1]}(0)=a_{1}$ and $\mathfrak{f} \in \operatorname{ker} A_{\max }$, where $\mathfrak{f}$ is the class from $L^{2}(W)$ generated by $f$. If Assumption 3.6 holds then similarly to the proof of Theorem 3.8 we get that $\operatorname{dim}$ ker $A_{\max }$ is isomorphic to $\mathbb{C}^{2}$. By the same argument, we get $\operatorname{ker} A_{\min }=\{0\}$. Now the statement of this theorem follows from Theorem 2.4.

Theorem 3.13. [25] The set of all self-adjoint extensions of the linear relation $A_{\min }$ is described by the boundary conditions

$$
\widetilde{A}=\left\{\left(\begin{array}{l}
\mathfrak{f} \\
\mathfrak{g}
\end{array}\right) \in A_{\max }: C \Gamma_{0}\left(\begin{array}{l}
\mathfrak{f} \\
\mathfrak{g}
\end{array}\right)+D \Gamma_{1}\left(\begin{array}{l}
\mathfrak{f} \\
\mathfrak{g}
\end{array}\right)=0\right\}
$$

where $C, D$ are complex valued $2 \times 2$ matrices such that

$$
\operatorname{det}\left(C C^{*}+D D^{*}\right) \neq 0, \quad C D^{*}=D C^{*} .
$$

In particular, linear relations $A_{0}$ and $A_{1}$ defined by equalities (2.11) are self-adjoint extensions of the linear relation $A_{\min }$. Extensions $A_{0}$ and 
$A_{1}$ corresponding to boundary triple (3.21) coincide with the Dirichlet extension and the Neumann extension

$$
\begin{aligned}
& A_{D}:=\left\{\left(\begin{array}{l}
\mathfrak{f} \\
\mathfrak{g}
\end{array}\right) \in A_{\max }: f(0)=f(l)=0\right\}, \\
& A_{N}:=\left\{\left(\begin{array}{l}
\mathfrak{f} \\
\mathfrak{g}
\end{array}\right) \in A_{\max }: f^{[1]}(0)=f^{[1]}(l)=0\right\},
\end{aligned}
$$

respectively.

\subsection{Functions $c(x, \lambda)$ and $s(x, \lambda)$. Weyl function of linear relation $A_{\max }$}

Let Assumption 2.10 and Assumption 3.6 hold. It follows from Theorem 2.9 that for each fixed $\lambda \in \mathbb{C}$ there exist unique vector-functions $\vec{c}(x, \lambda)$ and $\vec{s}(x, \lambda)$ satisfying the initial conditions

$$
\begin{array}{ll}
c(0, \lambda)=1, & c^{[1]}(0, \lambda)=0, \\
s(0, \lambda)=0, & s^{[1]}(0, \lambda)=1,
\end{array}
$$

such that the pairs $\{\vec{c}, \lambda c\}$ and $\{\vec{s}, \lambda s\}$ satisfy system (3.3). Here we have inclusions $c, s \in \mathcal{L}^{2}(W)$. Let $\mathfrak{c}(\lambda)$ and $\mathfrak{s}(\lambda)$ be classes from $L^{2}(W)$ generated by $c(x, \lambda)$ and $s(x, \lambda)$, respectively. Then

$$
\left(\begin{array}{c}
\mathfrak{c}(\lambda) \\
\lambda \mathfrak{c}(\lambda)
\end{array}\right),\left(\begin{array}{c}
\mathfrak{s}(\lambda) \\
\lambda \mathfrak{s}(\lambda)
\end{array}\right) \in A_{\max }
$$

It is known (see [6]) that functions $c(x, \lambda)$ and $s(x, \lambda)$ are entire in $\lambda$ of order not greater that $1 / 2$.

By conditions (3.33) functions $c$ and $s$ are linearly independent, and it follows from Assumption 3.6 that classes $\mathfrak{c}(\lambda)$ and $\mathfrak{s}(\lambda)$ are linearly independent too. Any element $\mathfrak{f}_{\lambda}$ from the defect subspace $\mathfrak{N}_{\lambda}$ can be represented as

$$
\mathfrak{f}_{\lambda}=a_{1} \mathfrak{c}(\lambda)+a_{2} \mathfrak{s}(\lambda), \quad a_{1}, a_{2} \in \mathbb{C} .
$$

Theorem 3.14. The generalized Wronskian of the functions $\vec{c}(x, \lambda)$ and $\vec{s}(x, \lambda)$ is a constant:

$$
[\vec{c}, \vec{s}]=c(x, \lambda) s^{[1]}(x, \lambda)-c^{[1]}(x, \lambda) s(x, \lambda)=1, \quad x \in[0, l] .
$$

Proof. Note that both pairs $\left(\begin{array}{c}\mathfrak{s} \\ \lambda \mathfrak{s}\end{array}\right)$ and $\left(\frac{\overline{\mathfrak{s}}}{\lambda \mathfrak{s}}\right)$ belong or do not belong to the linear relation $A_{\max }$ simultaneously. The application of Green's identity in the form (3.10) to pairs $\left(\begin{array}{c}\mathfrak{c} \\ \lambda \mathfrak{c}\end{array}\right)$ and $\left(\frac{\overline{\mathfrak{s}}}{\lambda \mathfrak{s}}\right)$ gives

$$
\left.[\vec{c}(t, \lambda), \vec{s}(t, \lambda)]\right|_{0} ^{x}=0 .
$$


Theorem 3.15. The Weyl function and the $\gamma$-field of the linear relation $A_{\max }$ corresponding to boundary triple $\left\{\mathbb{C}^{2}, \Gamma_{0}, \Gamma_{1}\right\}$ from (3.21) have the forms

$$
\begin{gathered}
M(\lambda)=\frac{-1}{s(l, \lambda)}\left(\begin{array}{cc}
c(l, \lambda) & -1 \\
-1 & s^{[1]}(l, \lambda)
\end{array}\right), \\
\gamma(\lambda)=\frac{1}{s(l, \lambda)}(\mathfrak{c}(\lambda) s(l, \lambda)-c(l, \lambda) \mathfrak{s}(\lambda) \quad \mathfrak{s}(\lambda)) .
\end{gathered}
$$

Proof. Let $\mathfrak{f}_{\lambda}=a_{1} \mathfrak{c}(\lambda)+a_{2} \mathfrak{s}(\lambda)$. Then

$$
\begin{gathered}
\Gamma_{0}\left(\begin{array}{c}
\mathfrak{f}_{\lambda} \\
\lambda \mathfrak{f}_{\lambda}
\end{array}\right)=\left(\begin{array}{cc}
1 & 0 \\
c(l, \lambda) & s(l, \lambda)
\end{array}\right)\left(\begin{array}{l}
a_{1} \\
a_{2}
\end{array}\right)=: Y_{0}\left(\begin{array}{c}
a_{1} \\
a_{2}
\end{array}\right), \\
\Gamma_{1}\left(\begin{array}{c}
\mathfrak{f}_{\lambda} \\
\lambda \mathfrak{f}_{\lambda}
\end{array}\right)=\left(\begin{array}{cc}
0 & 1 \\
-c^{[1]}(l, \lambda) & -s^{[1]}(l, \lambda)
\end{array}\right)\left(\begin{array}{l}
a_{1} \\
a_{2}
\end{array}\right)=: Y_{1}\left(\begin{array}{c}
a_{1} \\
a_{2}
\end{array}\right) .
\end{gathered}
$$

It follows from Definition 2.5 of the Weyl function and equality (3.36) that

$$
\begin{aligned}
M(\lambda)=Y_{1} Y_{0}^{-1}= & \frac{-1}{s(l, \lambda)}\left(\begin{array}{cc}
c(l, \lambda) & -1 \\
c^{[1]}(l, \lambda) s(l, \lambda)-c(l, \lambda) s^{[1]}(l, \lambda) & s^{[1]}(l, \lambda)
\end{array}\right) \\
& =\frac{-1}{s(l, \lambda)}\left(\begin{array}{cc}
c(l, \lambda) & -1 \\
-1 & s^{[1]}(l, \lambda)
\end{array}\right) .
\end{aligned}
$$

Finally, by definition of the $\gamma$-field we have

$$
\begin{gathered}
\gamma(\lambda)=(\mathfrak{c}(\lambda) \quad \mathfrak{s}(\lambda)) Y_{0}^{-1} \\
=\frac{1}{s(l, \lambda)}(\mathfrak{c}(\lambda) s(l, \lambda)-c(l, \lambda) \mathfrak{s}(\lambda) \quad \mathfrak{s}(\lambda)) .
\end{gathered}
$$

\section{Weyl functions of intermediate extensions of linear relation $A_{\min }$}

In this section the boundary triples and the corresponding Weyl functions for intermediate extensions of the linear relation $A_{\min }$ are constructed.

Definition 4.1. Let us set

$$
\begin{array}{ll}
A_{D 0}:=\left\{\left(\begin{array}{l}
\mathfrak{f} \\
\mathfrak{g}
\end{array}\right) \in A_{D}: f^{[1]}(0)=0\right\}, & A_{D l}:=\left\{\left(\begin{array}{l}
\mathfrak{f} \\
\mathfrak{g}
\end{array}\right) \in A_{D}: f^{[1]}(l)=0\right\}, \\
A_{N 0}:=\left\{\left(\begin{array}{l}
\mathfrak{f} \\
\mathfrak{g}
\end{array}\right) \in A_{N}: f(0)=0\right\}, & A_{N l}:=\left\{\left(\begin{array}{l}
\mathfrak{f} \\
\mathfrak{g}
\end{array}\right) \in A_{N}: f(l)=0\right\} .
\end{array}
$$


It follows from Definition 4.1, (3.31), and (3.32) that

$$
\begin{aligned}
& A_{D 0}:=\left\{\left(\begin{array}{l}
\mathfrak{f} \\
\mathfrak{g}
\end{array}\right) \in A_{\text {max }}: f(0)=f(l)=f^{[1]}(0)=0\right\}, \\
& A_{D l}:=\left\{\left(\begin{array}{l}
\mathfrak{f} \\
\mathfrak{g}
\end{array}\right) \in A_{\text {max }}: f(0)=f(l)=f^{[1]}(l)=0\right\}, \\
& A_{N 0}:=\left\{\left(\begin{array}{l}
\mathfrak{f} \\
\mathfrak{g}
\end{array}\right) \in A_{\text {max }}: f(0)=f^{[1]}(0)=f^{[1]}(l)=0\right\}, \\
& A_{N l}:=\left\{\left(\begin{array}{l}
\mathfrak{f} \\
\mathfrak{g}
\end{array}\right) \in A_{\text {max }}: f(l)=f^{[1]}(0)=f^{[1]}(l)=0\right\} .
\end{aligned}
$$

Theorem 4.2. Linear relation $A_{D 0}$ is symmetric in $L^{2}(W)$ with deficiency indices $(1,1)$ and the following conditions hold:

(i) The adjoint linear relation $A_{D 0}^{*}$ has the form

$$
A_{D 0}^{*}=\left\{\left(\begin{array}{l}
\mathfrak{f} \\
\mathfrak{g}
\end{array}\right) \in A_{\max }: f(l)=0\right\} .
$$

(ii) The tuple $\left\{\mathbb{C}, \Gamma_{0}^{D 0}, \Gamma_{1}^{D 0}\right\}$, where

$$
\Gamma_{0}^{D 0}\left(\begin{array}{l}
\mathfrak{f} \\
\mathfrak{g}
\end{array}\right)=f^{[1]}(0), \quad \Gamma_{1}^{D 0}\left(\begin{array}{l}
\mathfrak{f} \\
\mathfrak{g}
\end{array}\right)=-f(0),
$$

is a boundary triple for $A_{D 0}^{*}$.

(iii) The corresponding Weyl function and the $\gamma$-field have the form

$$
M_{D 0}(\lambda)=\frac{s(l, \lambda)}{c(l, \lambda)}, \quad \gamma_{D 0}(\lambda)=\mathfrak{s}(\lambda)-\frac{s(l, \lambda)}{c(l, \lambda)} \mathfrak{c}(\lambda)
$$

Proof. (i) Suppose $\left(\begin{array}{l}\mathfrak{f} \\ \mathfrak{g}\end{array}\right) \in A_{D 0}$. By definition $\left(\begin{array}{l}\mathfrak{u} \\ \mathfrak{v}\end{array}\right) \in A_{D 0}^{*}$ holds if and only if

$$
(\mathfrak{g}, \mathfrak{u})_{L^{2}(W)}=(\mathfrak{f}, \mathfrak{v})_{L^{2}(W)} .
$$

The last equality is equivalent to

$$
\left(\Gamma_{1}\left(\begin{array}{l}
\mathfrak{f} \\
\mathfrak{g}
\end{array}\right), \Gamma_{0}\left(\begin{array}{l}
\mathfrak{u} \\
\mathfrak{v}
\end{array}\right)\right)_{\mathcal{H}}=\left(\Gamma_{0}\left(\begin{array}{l}
\mathfrak{f} \\
\mathfrak{g}
\end{array}\right), \Gamma_{1}\left(\begin{array}{l}
\mathfrak{u} \\
\mathfrak{v}
\end{array}\right)\right)_{\mathcal{H}} .
$$


Since $f^{[1]}(l)$ is arbitrary, the last equality holds if and only if $u(l)=0$.

(ii) Let us show that Green's identity (in the sense of Definition 2.2) holds for the mappings $\Gamma_{0}^{D 0}, \Gamma_{1}^{D 0}$, which are defined on $A_{D 0}^{*}$. It is clear that $A_{D 0}^{*} \subset A_{\text {max }}$. Hence, for any $\left(\begin{array}{l}\mathfrak{f} \\ \mathfrak{g}\end{array}\right),\left(\begin{array}{l}\mathfrak{u} \\ \mathfrak{v}\end{array}\right) \in A_{D 0}^{*}$ the equality (3.13) holds and taking into account (4.5) we have

$$
(\mathfrak{g}, \mathfrak{u})_{L^{2}(W)}-(\mathfrak{f}, \mathfrak{v})_{L^{2}(W)}=f^{[1]}(0) \overline{u(0)}-f(0) \overline{u^{[1]}(0)} .
$$

It remains to check that the mapping $\Gamma_{D 0}=\left(\begin{array}{l}\Gamma_{0}^{D 0} \\ \Gamma_{1}^{D 0}\end{array}\right): A_{D 0}^{*} \rightarrow \mathbb{C} \oplus \mathbb{C}$ is surjective, which follows directly from the subjectivity of the mapping $\Gamma$ on $A_{\max }$.

(iii) The defect subspace of linear relation $A_{D 0}^{*}$ has the form

$$
\mathfrak{N}_{\lambda}\left(A_{D 0}^{*}\right)=\operatorname{span}\{\mathfrak{c}(\lambda)+k \mathfrak{s}(\lambda)\}
$$

where the coefficient $k$ is chosen to satisfy $f_{\lambda}(l)=0$. Further

$$
\Gamma_{0}^{D 0} \hat{f}_{\lambda}=k=-\frac{c(l, \lambda)}{s(l, \lambda)}, \quad \Gamma_{1}^{D 0} \hat{f}_{\lambda}=-1,
$$

and finally

$$
M_{D 0}(\lambda)=\frac{s(l, \lambda)}{c(l, \lambda)}, \quad \gamma_{D 0}(\lambda)=\mathfrak{s}(\lambda)-\frac{s(l, \lambda)}{c(l, \lambda)} \mathfrak{c}(\lambda) .
$$

Similar theorems for extensions $A_{D l}, A_{N 0}$, and $A_{N l}$ are given below without proofs.

Theorem 4.3. Linear relation $A_{D l}$ is symmetric in $L^{2}(W)$ with deficiency indices $(1,1)$, and the following conditions hold:

(i) The adjoint linear relation $A_{D l}^{*}$ has the form

$$
A_{D l}^{*}=\left\{\left(\begin{array}{l}
\mathfrak{f} \\
\mathfrak{g}
\end{array}\right) \in A_{\max }: f(0)=0\right\} .
$$

(ii) The tuple $\left\{\mathbb{C}, \Gamma_{0}^{D l}, \Gamma_{1}^{D l}\right\}$, where

$$
\Gamma_{0}^{D l}\left(\begin{array}{l}
\mathfrak{f} \\
\mathfrak{g}
\end{array}\right)=f^{[1]}(l), \quad \Gamma_{1}^{D l}\left(\begin{array}{l}
\mathfrak{f} \\
\mathfrak{g}
\end{array}\right)=f(l),
$$

is a boundary triple for $A_{D l}^{*}$. 
(iii) The corresponding Weyl function and the $\gamma$-field have the form

$$
M_{D l}(\lambda)=\frac{s(l, \lambda)}{s^{[1]}(l, \lambda)}, \quad \gamma_{D l}(\lambda)=\frac{\mathfrak{s}(\lambda)}{s^{[1]}(l, \lambda)} .
$$

Theorem 4.4. Linear relation $A_{N 0}$ is symmetric in $L^{2}(W)$ with deficiency indices $(1,1)$ and the following conditions hold:

(i) The adjoint linear relation $A_{N 0}^{*}$ has the form

$$
A_{N 0}^{*}=\left\{\left(\begin{array}{l}
\mathfrak{f} \\
\mathfrak{g}
\end{array}\right) \in A_{\max }: f^{[1]}(l)=0\right\} .
$$

(ii) The tuple $\left\{\mathbb{C}, \Gamma_{0}^{D l}, \Gamma_{1}^{D l}\right\}$, where

$$
\Gamma_{0}^{N 0}\left(\begin{array}{l}
\mathfrak{f} \\
\mathfrak{g}
\end{array}\right)=f^{[1]}(0), \quad \Gamma_{1}^{N 0}\left(\begin{array}{l}
\mathfrak{f} \\
\mathfrak{g}
\end{array}\right)=-f(0),
$$

is a boundary triple for $A_{N 0}^{*}$.

(iii) The corresponding Weyl function and $\gamma$-field have the form

$$
M_{N 0}(\lambda)=\frac{s^{[1]}(l, \lambda)}{c^{[1]}(l, \lambda)}, \quad \gamma_{N 0}(\lambda)=\mathfrak{s}(\lambda)-\frac{s^{[1]}(l, \lambda)}{c^{[1]}(l, \lambda)} c(\cdot, \lambda) .
$$

Theorem 4.5. Linear relation $A_{N l}$ is symmetric in $L^{2}(W)$ with deficiency indices $(1,1)$ and the following conditions hold:

(i) The adjoint linear relation $A_{N l}^{*}$ has the form

$$
A_{N 1}^{*}=\left\{\left(\begin{array}{l}
\mathfrak{f} \\
\mathfrak{g}
\end{array}\right) \in A_{\max }: f^{[1]}(0)=0\right\} .
$$

(ii) The tuple $\left\{\mathbb{C}, \Gamma_{0}^{N l}, \Gamma_{1}^{N l}\right\}$ where

$$
\Gamma_{0}^{N l}\left(\begin{array}{l}
\mathfrak{f} \\
\mathfrak{g}
\end{array}\right)=f^{[1]}(l), \quad \Gamma_{1}^{N l}\left(\begin{array}{l}
\mathfrak{f} \\
\mathfrak{g}
\end{array}\right)=f(l)
$$

is a boundary triple for $A_{N l}^{*}$.

(iii) The corresponding Weyl function and $\gamma$-field have the form

$$
M_{N l}(\lambda)=\frac{c(l, \lambda)}{c^{[1]}(l, \lambda)}, \quad \gamma_{N l}(\lambda)=\frac{c(\cdot, \lambda)}{c^{[1]}(l, \lambda)} .
$$

Remark 4.6. The Weyl functions $M_{D 0}, M_{N 0}$ in the case $d Q \equiv 0$ coincide with the functions $\Omega_{0}, \Omega_{1}$, see [18, p. 666, (2.40-41)]. 


\section{Special cases}

\subsection{Absolutely continuous case. Sturm-Liouville operator}

Let functions $P, Q$ and $W$ be absolutely continuous on $[0, l]$, i.e. there exist functions $p, q$ and $w$ from $L^{1}[0, l]$ such that

$$
P(x)=\int_{0}^{x} p(t) d t, \quad Q(x)=\int_{0}^{x} q(t) d t, \quad W(t)=\int_{0}^{x} w(t) d t,
$$

$p(t) \neq 0$ and $w(t) \geq 0$ almost everywhere with respect to Lebesgue measure on $[0, l]$. In addition, we require that the space $L^{2}(W)$ be nontrivial. The last requirement is equivalent to $W(l)>W(0)$.

In this special case system (1.1) can be written in the form

$$
J \vec{f}^{\prime}(x)=\lambda H(x) \vec{f}(x)+V(x) \vec{f}(x), \quad \vec{f}(0)=\vec{a}(0),
$$

where

$$
H(x)=\left(\begin{array}{cc}
w(x) & 0 \\
0 & 0
\end{array}\right), \quad V(x)=\left(\begin{array}{cc}
-q(x) & 0 \\
0 & p(x)
\end{array}\right), \quad \vec{f}(x)=\left(\begin{array}{c}
f(x) \\
f^{[1]}(x)
\end{array}\right)
$$

or, equivalently,

$$
\left\{\begin{array}{l}
-\left(f^{[1]}\right)^{\prime}(x)=\lambda w(x) f(x)-q(x) f(x), \\
f^{\prime}(x)=p(x) f^{[1]}(x) .
\end{array}\right.
$$

System (5.3) is equivalent to the Sturm-Liouville equation (see [26])

$$
-\frac{d}{d x}\left(\frac{1}{p(x)} \frac{d}{d x} f(x)\right)+q(x) f(x)=\lambda w(x) f(x) .
$$

with the initial conditions

$$
f(0)=a_{1}, \quad f^{[1]}(0)=a_{2} .
$$

More general canonical systems (5.2) were studied in [16, 20,24], where, in particular, it was shown that the maximal and the minimal operators associated with such canonical systems can be linear relations with nontrivial multivalued part. In the 2-dimensional case the multivalued part of the maximal operator was calculated explicitly in terms of the so called $H$-indivisible intervals, [16]. Actually in the absolutely continuous case the results of the paper can be easily derived from the results of [4] and [23]. 


\subsection{Discrete case. Stieltjes string}

Let us consider system (3.4) in the case $d Q \equiv 0, d P=d x$, and $W$ be a left-continuous monotonically nondecreasing piecewise constant function on $[0, l]$ that has at least two growth points. Let $\left\{x_{j}\right\}_{j=0}^{n-1}$ be the growth points of $W$ such that

$$
0=x_{0}<x_{1}<\ldots<x_{n-1}<x_{n}:=l .
$$

By $w_{j}$ denote

$$
w_{j}:=W\left(x_{j}+0\right)-W\left(x_{j}\right) \quad(j \in\{1,2, \ldots, n-1\}) .
$$

The distance between neighboring growth points is denoted by

$$
l_{j}:=x_{j}-x_{j-1} \quad(j \in\{1,2, \ldots, n\}) .
$$

Finally for convenience denote

$$
f_{j}:=f\left(x_{j}\right), \quad g_{j}:=g\left(x_{j}\right), \quad h_{j}:=f^{[1]}\left(x_{j}\right) .
$$

With generating function $W$ the space $L^{2}(W)$ is isomorphic to $\mathbb{C}^{n}$, and each of its element is a vector $\left[f_{0}, f_{1}, \ldots, f_{n-1}\right]^{T}$.

It is easy to check that by the above assumptions one can choose closed on the left intervals $i_{1}, i_{2}$ such that they satisfy Proposition 3.7. For instance, it is sufficient to choose intervals $i_{j}$ with the only growth point $x_{j-1}(j \in\{1,2\})$. Theh the spaces $L^{2}\left(i_{j}, W\right)$ obviously are nontrivial and inequality (3.16) takes the following form

$$
\frac{w_{1} l_{1}}{w_{2}}>0
$$

Combining (5.6), (5.7), and (5.8) we can rewrite system (3.4) as

$$
\left\{\begin{array}{l}
f_{j+1}-f_{j}=h_{j+1} l_{j+1}, \\
h_{j+1}-h_{j}=-w_{j} g_{j}
\end{array}\right.
$$

where $j \in\{0,1, \ldots, n-1\}$.

Proposition 5.1. In the assumptions of case 5.2 the multivalued part of the linear relation $A_{\max }$ has the form

$$
\left\{\left(c_{1}, 0,0, \ldots, 0, c_{2}\right)^{T}: c_{1}, c_{2} \in \mathbb{C}\right\}
$$

and the linear relation $A_{\min }$ is the graph of a single-valued linear operator. 
Proof. Let $\mathfrak{f}$ be the zero element of $L^{2}(W)$. Thus, in (5.10) we have $f_{j}=0$ as $j \in\{0,1, \ldots, n-1\}$, hence $h_{j}=0$ as $j \in\{1,2, \ldots, n-1\}$ and $g_{j}=0$ as $j \in\{1,2, \ldots, n-2\}$. The converse is also true: since we can choose $h_{0}=w_{0} g_{0}$ then for each vector $\mathfrak{g} \in L^{2}(W)$ of the form (5.11) the pair $\left(\begin{array}{l}0 \\ \mathfrak{g}\end{array}\right)$ belongs to the linear relation $A_{\max }$.

If, in addition, $f_{n}=h_{0}=h_{n}=0$ then it follows from (5.10) that $g_{j}=0$ as $j \in\{0,1, \ldots n-1\}$.

\subsection{Mixed case. Krein-Feller string}

A more general case can be obtained if we suppose $d Q \equiv 0, d P=d x$ and $W$ is an arbitrary mototonically nondecreasing function.

In this Proposition 3.7 holds if and only if $W$ has at least two distinct growth points on $[0, l]$ :

$$
0<W\left(x_{0}\right)<W\left(x_{1}\right) \leq W(l) .
$$

Now system (3.4) has the following form:

$$
\left\{\begin{array}{l}
f(x)-f(0)=\int_{0}^{x} f^{[1]}(t) d t, \\
f^{[1]}(x)-f^{[1]}(0)=-\int_{0}^{x} g(t) d W(t) .
\end{array}\right.
$$

In particular we have the next

Proposition 5.2. Suppose the assumptions of case 5.3 are satisfied. If a $\operatorname{pair}\left(\begin{array}{l}\mathfrak{f} \\ \mathfrak{g}\end{array}\right) \in L^{2}(W)^{2}$ belongs to linear relation $A_{\max }$ then there exists $f \in \mathfrak{f}$ such that $f$ is absolutely continuous with respect to Lebesgue measure and its derivative coincides with $f^{[1]}$ almost everywhere.

Let us rewrite system (5.13) as

$$
f(x)=f(0)+x f^{[1]}(0)-\int_{0}^{x}\left(\int_{0}^{t} g(s) d W(s)\right) d t .
$$

The function $\int_{0}^{t} g(s) d W(s)$ is left-continuous and of bounded variation on $[0, l]$. It follows from (2.14) that equality (5.14) can be rewritten as

$$
f(x)=f(0)+x f^{[1]}(0)-\int_{0}^{x}(x-s) g(s) d W(s) .
$$


Definition 5.3. [17] A function $f$ is said to belong to the Stieltjes class $S^{+}$if $f \in \mathrm{R}$ and $f$ admits a holomorphic non-negative continuation to $(-\infty, 0)$.

In the paper [18] the differential operation defined by (5.15) was investigated. I. S. Kats and M. G. Krein showed that under assumptions of the case 5.2 the Weyl functions $M_{D 0}, M_{N 0}$ and $M_{N l}$ constructed in section 4, belong to the Stieltjes class $S^{+}$, see [18, p. 666, Lemma 2.3].

\section{References}

[1] R. Arens, Operational calculus of linear relations // Pacific Journal of Mathematics, 11 (1961), No. 1, 9-23.

[2] D.Z. Arov, H. Dym, Bitangential Direct and Inverse Problems for Systems of Integral and Differential Equations, 2012.

[3] F. V. Atkinson, Discrete and continuous boundary problems, Academic Press, New York-London, Mathematics in Science and Engineering, Vol. 8, 1964.

[4] J. Behrndt, S. Hassi, H. de Snoo, R. Wietsma, Square-integrable solutions and Weyl functions for singular canonical systems // Mathematische Nachrichten, 284 (2011), No. 11-12, 1334-1384.

[5] C. Bennewitz, Symmetric relations on a Hilbert space, Lecture Notes in Math., Springer, Berlin, Heidelberg, 280 (1972), 212-218.

[6] C. Bennewits, Spectral Asymptotics for Sturm-Liouville Equations // Proceedings of the London Mathematical Society, (3) 59 (1989), No. 2, 294-338.

[7] V.M. Bruk, On a class of problems with the spectral parameter in the boundary conditions // Mat. Sb., 100 (1976), 210-216.

[8] J.W. Calkin, Abstract symmetric boundary conditions // TAMS, 45 (1939), No. 3, 369-442.

[9] V. Derkach, S. Hassi, M. Malamud, H. de Snoo, Boundary relations and their Weyl families // Transactions of the American Mathematical Society, 358 (2006), No. 12, 5351-5400.

[10] V.A. Derkach, M. M. Malamud, Generalized resolvents and the boundary value problems for hermitian operators with gaps // J. Funct. Anal., 95 (1991), 1-95.

[11] V.A. Derkach, M. M. Malamud, The extension theory of hermitian operators and the moment problem // J. Math. Sciences, 73 (1995), 141-242.

[12] V.A. Derkach, M. M. Malamud, Extension theory of symmetric operators and boundary value problems, Proceedings of Institute of Mathematics NAS of Ukraine, Kyiv, 2017, 573 p. (2017)

[13] W. Feller, On Second Order Differential Operators // Annals of Mathematics, 61 (1955), No. 1, 90-105. 
[14] V.I. Gorbachuk, M.L. Gorbachuk, Boundary problems for differential operator equations, Naukova Dumka, Kiev, 1984.

[15] E. Hewitt, Integration by Parts for Stieltjes Integrals // The American Mathematical Monthly, 67 (1960), No. 5, 419-423.

[16] I. S. Kac, Linear relations generated by a canonical differential equation of phase dimension 2 and decomposability in eigenfunctions // Algebra i analiz, 14 (2002), No. 3, 86-120.

[17] I. S. Kac, M. G. Krein, R-functions - analytic functions mapping the upper half plane into itself, Supplement I to the Russian translation of F. V. Atkinson, Discrete and continuous boundary problems, Mir, Moscow, 1968, 629-647.

[18] I. S. Kac, M. G. Krein, On the spectral functions of the string, Supplement II to the Russian translation of F. V. Atkinson, Discrete and continuous boundary problems, Mir, Moscow, 1968, 648-737.

[19] A. N. Kochubey, On extensions of symmetric operators and symmetric binary relations // Math. Notes, 17 (1975), No. 1, 41-48.

[20] M. Lesch, M. Malamud, On the deficiency indices and self-adjointness of symmetric Hamiltonian systems //, J. Differential Equations, 189 (2003), No. 2, 556-615

[21] M. M. Malamud, On the formula of generalized resolvents of a nondensely defined Hermitian operator // Ukr. Mat. Zh., 44 (1992), No. 12, 1658-1688.

[22] V. Mogilevsky, Boundary triplets and Titchmarsh - Weyl functions of differential operators with arbitrary deficiency indices // Methods of Funct. Anal. Topology, 15 (2009), No. 3, 280-300.

[23] V.I. Mogilevskii, Spectral and pseudospectral functions of Hamiltonian systems: development of the results by Arov-Dym and Sakhnovich // Methods of Funct. Anal. Topology, 21 (2015), No. 4, 370-402.

[24] B. Orcutt, Canonical differential equations, Ph.D. thesis, University of Virginia, 1969.

[25] F.S. Rofe-Beketov, On selfadjoint extensions of differential operators in a space of vector-functions // Teor. Funkts., Funkts. Anal. i Prilozhen., 8 (1969), 3-24.

[26] E. C. Titchmarsh, Eigenfunction expansions associated with second order differential equations, Part I, 2nd edn (Oxford University Press, 1962).

\section{CONTACT INFORMATION}

Dmytro Strelnikov Vasyl' Stus Donetsk National University, Vinnitsya, Ukraine

E-Mail: d.strelnikov@donnu.edu.ua 